Meta

Journal des tradlucteurs

Translators' Journal

\title{
Poésie : le sens en effet
}

Étude d'un translème

\section{Annie Brisset}

Volume 29, numéro 3, septembre 1984

URI : https://id.erudit.org/iderudit/002148ar

DOI : https://doi.org/10.7202/002148ar

Aller au sommaire du numéro

Éditeur(s)

Les Presses de l'Université de Montréal

ISSN

0026-0452 (imprimé)

1492-1421 (numérique)

Découvrir la revue

Citer cet article

Brisset, A. (1984). Poésie : le sens en effet : étude d'un translème. Meta, 29(3),

259-266. https://doi.org/10.7202/002148ar

Ce document est protégé par la loi sur le droit d'auteur. L'utilisation des services d'Érudit (y compris la reproduction) est assujettie à sa politique d'utilisation que vous pouvez consulter en ligne.

https://apropos.erudit.org/fr/usagers/politique-dutilisation/
Cet article est diffusé et préservé par Érudit.

Érudit est un consortium interuniversitaire sans but lucratif composé de l'Université de Montréal, l'Université Laval et l'Université du Québec à Montréal. Il a pour mission la promotion et la valorisation de la recherche. https://www.erudit.org/fr/ 


\section{POÉSIE : LE SENS EN EFFET ${ }^{\star}$ ÉTUDE D'UN TRANSLÈME}

ANNIE BRISSET

Une conception largement répandue parmi les praticiens et les théoriciens réduit le texte poétique à un contenu sémantique d'un intérêt cognitif secondaire, de sorte que son unique fonction consisterait à produire un effet esthétique sur le récepteur. Héritière de l'ancienne rhétorique, cette notion d'effet esthétique, ou poétique, s'apparente à ce qu'on appelait l'ornatus, en ce sens que la poésie se différencierait des autres types de discours sur la présence d'un supplément ornemental, étranger à la signification du texte. Derrière cette conception de la poésie, se profile, avec certaines modifications, l'ancienne dichotomie du fond et de la forme, à laquelle correspond cette dissociation hiérarchisée : traduire le sens, traduire le style - selon la célèbre formule de Charles Taber et d'Eugène Nida.

Or, les acquis de la théorie du texte et du discours nous autorisent à rejeter cette conception, qui repose sur l'idéologie mystifiante du "beau " en poésie, c'est-à-dire sur un apriorisme qui valorise, jusqu'à le sublimer, tout énoncé portant les marques conventionnelles de la poésie ${ }^{1}$. Cette qualité auratique que l'on prête à l'écriture est d'ailleurs démentie par les poètes eux-mêmes : Khlebnikov, Huidobro, Pessoa, Antonin Artaud, Auden ou Paul Celan et, plus près de nous, C. Milosz, Michel Deguy ou François Charron,pour ne citer que quelques-uns de ceux qui appartiennent au champ de notre modernité. De plus, l'analyse de leurs cuvres permet d'affirmer que le texte poétique est une totalité signifiante dans la mesure où il est énonciation, travail du sujet sur la langue et procès de signifiance, dans la mesure où il se présente aussi comme un système de modélisations secondaires qui se chevauchent et s'interpénètrent ${ }^{2}$.

* COLLOQUE DE GLENDON, 1980.

1. Dans son analyse critique de la traduction de Strette de Paul Celan par A. du Bouchet, J. Daive et J.-P. Burgart, Meschonnic montre de façon convaincante à quoi aboutit cette idéologie lorsqu'elle préside à la traduction : " L'idéologie poétique a horreur du mot simple. Elle se plaît aux mots qu'Yves Bonnefoy appelle des mots de l'aspect. Elle fuit le terme générique d'une série. Elle montre sa culture et sa recherche. Toutes les perles de l'école sont à son collier. Pas habiter mais avoir logis. Pas pensées mais pensers. Pas tomber mais verser. Pas oblique, non déclive... Pas dormir pour Schlafen, mais sommeiller... " Un peu plus tôt, Meschonnic disait : " Le rapport entre une idéologie de la poésie et la traduction de la poésie a été tel, ici, que la poésie traduite est devenue cette idéologie de la poésie. Au lieu que l'écriture transforme l'idéologie, c'est l'idéologie en langue d'arrivée qui a transformé l'écriture de la langue de départ... Le produit est une sous-littérature... Mais cela n'est pas Celan. " Henri Meschonnic (1973) : "On appelle cela traduire Celan ", dans Pour la poétique II, Paris, Gallimard, p. 402 et pp. 384-385.

2. Cette conception du texte comme énonciation et productivité, travail du sujet sur la langue et procès de signifiance se trouve exposée chez J. Kristeva (1969), notamment dans son essai intitulé : « Le texte et sa science ", dans Sèmeiotikè. Recherches pour une sémanalyse, Paris, Seuil, pp. 9-28. Dans la Structure du texte artistique, Paris, Gallimard, Lotman (1973), pour sa part, fait reposer la spécificité du texte (artistique) sur ce qu'il appelle la modélisation secondaire : " Les systèmes modélisants secondaires représentent des structures à la base desquelles se trouve la langue naturelle. Cependant, ultérieurement, le système reçoit une structure complémentaire secondaire de type idéologique, éthique, artistique ou de tout autre type. Les significations de ce système secondaire peuvent s'organiser selon des moyens propres 
Je vais tenter d'illustrer ce propos en prenant pour objet d'analyse un poème d'effet que son sens dénotatif ou référentiel semble rejeter précisément dans la catégorie du nonsense, pour en faire ce qu'Hélène Cixous appellerait un "poème sans sens ${ }^{3}$ ". Et, qui plus est, je vais m'en tenir à ce que ce poème a de plus spectaculaire, c'est-à-dire à l'ensemble des paronomases :

' reader'/" rider, 'fearer'/" farer', ' horror'/" hearer'

'O where are you going?' said reader to rider,

'That valley is fatal when furnaces burn,

Yonder's the midden whose odours will madden,

That gap is the grave where the tall return.'

' $O$ do you imagine,' said fearer to farer,

'That dusk will delay on your path to the pass,

Your diligent looking discover the lacking

Your footsteps feel from granite to grass?'

' $O$ what was that bird', said horror to hearer

'Did you see that shape in the twisted trees?

Behind you swiftly the figure comes softly,

The spot on your skin is a shocking disease.'

'Out of this house' - said rider to reader,

'Yours never will' - said farer to fearer,

'They're looking for you' - said hearer to horror,

As he left them there, as he left them there4.

Ces six lexèmes se détachent du texte pour des raisons qui sont avant tout d'ordre phonétique. Ils constituent un ensemble de paires lexicales dont les composants sont homophones, à un élément près. Selon le principe de G.M. Hopkins repris par R. Jakobson, la quasi-identité des signifiants appelle un rapprochement des signifiés ${ }^{5}$. En l'occurrence, le rapprochement sémantique des six termes s'impose doublement :

1. Ils comportent le même suffixe, qui est la marque d'un sème nucléaire comme désignant l'agent d'une action. 'Horror' est dépourvu de ce suffixe agentif mais si l'on considère qu'il s'agit d'une personnification, il a, comme les autres termes, le statut d'ac$\tan t^{6}$.

aux langues naturelles et selon des moyens d'autres systèmes sémiotiques. "Voir p. 71. Dans Linguistique et poétique, Paris, Larousse, D. Delas et J. Filliolet (1973) définissent le texte comme totalisation en fonctionnement ; cf. p. 46.

3. Edward Lear (1974) : Poèmes sans sens, Paris, Aubier-Flammarion, p. 58.

4. W.H. Auden (1932) : The Orators, Londres, Faber \& Faber.

5. Roman Jakobson (1963) : "Linguistique et poétique ", in Essais de linguistique générale, Paris, Minuit, pp. 235-236.

6. "L'actant peut être conçu comme celui qui accomplit ou qui subit l'acte indépendamment de toute autre détermination. Ainsi, pour citer Tesnière, à qui ce terme est emprunté, ' les actants sont les êtres ou les choses qui, à un titre quelconque et de quelque façon que ce soit, même au titre de simples figurants et de la façon la plus passive, participent au procès'. Dans cette perspective l'actant désignera un type d'unité syntaxique, de caractère proprement formel, antérieurement à tout investissement sémantique et/ou idéologique. " Dans J. Courtès et A.J. Greimas (1979) : Sémiotique. Dictionnaire raisonné de la théorie du langage, Paris, Hachette, p. 3. 
2. Ils se définissent aussi comme actants par rapport à un " dire " dont ils sont destinateurs ou destinataires; la marque de ce dire étant le verbum dicendi "said".

Je commencerai par analyser ce qui rapproche ou oppose ces six actants dans la modalité du "faire " en m'interrogeant sur la nature de l'action qu'ils désignent. Une analyse componentielle fait apparaître certaines constantes et certaines oppositions paradigmatiques, qui sont, pour la série 'reader'/'fearer'/ horror' activité mentale ou affect

intériorité statisme

et pour la série 'rider'/'farer'/" hearer' : activité physique extériorité dynamisme

Ces deux séries seront respectivement appelées paradigme des actants statiques et paradigme des actants dynamiques.

Première constatation : 'hearer' ne possède aucun des sèmes caractéristiques du paradigme où il est inscrit. Il possède en revanche ceux du paradigme opposé. Deuxième constatation : si l'on approfondit la nature de l'activité propre à chacun des actants, on s'aperçoit que les deux paradigmes sont hiérarchiquement structurés :

1. Le paradigme des actants statiques manifeste la disparition progressive d'une activité donnée au départ comme rationnelle (read).

2. Le paradigme des actants dynamiques manifeste la disparition progressive d'une activité donnée au départ comme physique (ride).

D'où il ressort que les trois termes de chaque paradigme peuvent représenter trois phases ou trois degrés d'une même activité ; ensuite, ces trois phases sont, dans leur progression, en correspondance terme à terme :

\begin{tabular}{cccc}
\hline actant statique & rationalité & actant dynamique & mobilité \\
\hline reader & ++ & rider & ++ \\
fearer & + & farer & + \\
horror & $\varnothing$ & hearer & $\varnothing$ \\
\hline
\end{tabular}

Une double superposition ou réduction sémantique est alors envisageable :

1. Réduction verticale - Les trois actants de chaque paradigme se confondent en un seul dans la mesure où ils se différencient par le degré d'une activité et non par la nature de cette activité. Par cette réduction on obtient, de part et d'autre, non plus trois actants mais trois variantes du même actant. Il est entendu que la réduction est réalisable du point de vue des connotations fournies par le contexte (signifiance) et non du point de vue dénotatif (signification) et que l'équivalence repose sur l'assimilation de la partie au tout, en l'occurrence d'un sème à un sémème.

2. Réduction horizontale - Chaque actant se confond avec ce qui est à la fois son double, phonétique ou sémantique, ou encore son complément sémantique. Par exemple, on peut considérer 'reader' et 'rider' comme l'illustration des deux versants complémentaires de l'activité humaine, intellectuelle et physique. Signalons au passage la superposition phonétique de 'rider' ('raidə/) et de 'writer' (/'raitə/) qui n'a sûrement pas échappé à Auden, jongleur de mots et poète du " wit ", superposition qui maintient la relation complémentaire. 
Il existe une autre hiérarchisation des paradigmes, mais cette fois en relation inverse. Un sème est réitéré dans deux seulement de leurs constituants ; c'est, du côté des actants statiques, le sème " peur » (présent dans 'fearer' et 'horror') et, du côté des actants dynamiques, celui de la "mobilité " ou du déplacement (présent dans 'rider' et 'farer'). Si l'on compare les paradigmes, on voit que la peur croît en intensité, passant de 'fearer' à 'horror', en même temps que décroît la mobilité, passant de 'rider' à 'farer'.

\begin{tabular}{cccc}
\hline destinateur & peur & destinataire & mobilité \\
\hline reader & $\varnothing$ & rider & ++ \\
fearer & + & farer & + \\
horror & ++ & hearer & $\varnothing$ \\
\hline
\end{tabular}

Nouvelle constatation : 'reader' et ' hearer' ne sont indexés ni sur l'isotopie de la peur ni sur celle de la mobilité. Ils peuvent donc être vus comme le degré zéro de leur paradigme respectif. De plus, ils sont topologiquement codés puisqu'ils occupent dans le texte une position diamétralement opposée. Ils forment donc un nouveau couple d'actants. Ceuxci encadrent les trois autres et sont unis comme eux dans un rapport d'identité et de complémentarité sémantiques par rapport à un "dire", cette fois implicite et métaphorique, communiqué sous la forme d'une allégorie, ou plus exactement d'une fable.

Je m'explique : premier actant du poème, 'reader', qui désigne nommément le lecteur, fait de ce dernier la figure nécessaire du destinataire. De même, le verbum dicendi ('said') fonctionne comme opérateur métalinguistique et renvoie, comme le vers conclusif du poème, à une instance énonciative, à un observateur extérieur, destinateur du texte. Le poème réalise ainsi une mise en abyme de la lecture en ce sens qu'il constitue un message adressé par le poète à son lecteur et que ce message est communiqué sous la forme d'une fable, composée elle-même d'une série d'infra-messages, en l'occurrence d'une série de questions et de réponses échangées entre une double série ordonnée d'actants.

Dès l'instant où le lecteur se reconnaît en ' reader', actant de la fable, il devient à la fois lecteur-sujet et lecteur-objet, auditeur (' hearer') et protagoniste de la parabole qui lui est racontée. Par le biais des propos qu'on lui prête ("... said reader"), il est mis encore une fois en situation de réceptivité, ou plus exactement en situation d'écoute. Il est donc amené à s'identifier directement à l'actant 'hearer'. Autrement dit, 'reader' et 'hearer' sont interchangeables.

Par ailleurs, le jeu de la ressemblance et de la différence entre les actants de la fable racontée par le poème est un ressort de la dialectisation du message, dialectisation introduite par l'interpellation du lecteur. Manipulé, celui-ci doit en effet se reconnaître tour à tour dans la série des actants statiques et dans celle des actants dynamiques et faire siens leurs propos successifs. En ce sens, le dialogue des actants, qui est aussi le dialogue du lecteur avec son double, constitue une maïeutique animée par le poète. Mais quel est, brièvement, l'objet de ce débat singulier?

Dans ce poème, il existe une coréférentialité entre chaque locuteur et son registre délocutif ${ }^{7}$. Le premier registre, qui correspond aux actants statiques et qui s'étend sur les trois premières strophes, manifeste une répétitivité des structures phonétiques, syn-

7. La notion de registre délocutif, qui vient de Damourette et Pichon, est définie de manière simple par Ortigues : " Les signifiants de la personne se répartissent suivant un double registre d'oppositions distinctives : 
taxiques et sémantiques. Fixé dans les mêmes schémas formels, ce dire non évolutif repose sur une série d'oppositions qui se répètent invariablement :

fixité/sécurité/vérité vs changement/danger/erreur

Le registre des actants dynamiques, qui couvre les trois premiers vers de la quatrième strophe, en est l'antithèse. Si le discours des actants statiques est fixé dans une armature formelle et sémantique immuable, celui des actants dynamiques est au contraire imprévisible. À la rhétorique explicite des actants statiques répond la parole concise, incisive et elliptique des actants dynamiques. C'est, à la limite, la négation d'un discours, celuici n'étant exprimé qu'en fonction de l'action dont il affirme la suprématie. Action de rupture par rapport au lieu du discours ("Out of this house "), qui est lieu de fixité, la parole du narrateur venant alors relayer celle du dernier actant dynamique comme pour une prise en charge de son acte ("As he left them there...").

L'ensemble des actants de même que la sérialisation spatiale (I : 'valley', II : 'pass', III : 'spot') et la sérialisation temporelle se résorbent en une déixis : je (c'est-àdire moi, lecteur) - ici - maintenant, déixis qui se situe entre deux pôles, statisme et dynamisme, dont la valeur respective est mise en cause. Le maintien de la déixis ou, pour être plus explicite, le maintien du statu quo est opposé à son dépassement.

Le système actantiel ainsi dégagé aboutit à une interprétation générale du poème, interprétation à la fois noologique et cosmologique.

Du point de vue noologique, le texte met en scène les tensions existentielles d'une conscience individuelle partagée entre la crainte du changement et le désir d'une vie nouvelle rompant avec un univers figé, inscrit par conséquent dans la mort. Cette conscience est également divisée entre les forces inhibantes de la raison, incarnées par les actants statiques, et les forces agissantes du corps, qui poussent à la réalisation des impulsions et des désirs et auxquelles renvoient les actants dynamiques.

Du point de vue cosmologique, l'individu ('he') est opposé à une collectivité ('them') représentée par le paradigme des actants statiques et que l'on peut réduire à une catégorie particulière : la bourgeoisie, les intellectuels, les éducateurs ou même l'ordre établi, compte tenu des corrélations sémantiques entre les deux paradigmes d'actants. Quel que soit le sens particulier qu'on attribue à ces paradigmes en fonction des données socio-historiques, politiques ou biographiques qui entourent le texte, la polarité statique et dynamique demeure inchangée de même que la problématique de l'évolution. L'individu remet en cause une société figée, installée dans une fausse sécurité et dotée d'une fausse conscience.

Voilà un aperçu très schématique d'un sens possible du poème construit à partir du système actantiel que forment les trois paires paronomasiques ${ }^{8}$.

Je voudrais introduire ici la notion de translème. J'appelle translème une unité de traduction à caractère sémiotique car il s'agit d'une unité-système composée d'éléments significativement solidaires. Autrement dit, un translème est un réseau de relations qui unissent, dans un rapport sémantique, un ensemble d'éléments appartenant soit au plan

- l'opposition entre la personne qui parle (Je) et la personne à qui l'on s'adresse (Tu) constitue le registre de la communication ou de l'interlocution ;

- cette corrélation de la personnalité est opposable à son tour à la catégorie de la chose dont on parle (il) qui constitue le registre délocutif. "Edmond Ortigues (1962) : le Discours et le symbole, Paris, AubierMontaigne, p. 151.

8. Pour une analyse très détaillée de ce poème, voir A. Brisset (1980) : Structures de signifiance et traduction. Réflexions sur un poème de W.H. Auden, thèse déposée à l'Université d'Ottawa, $230 \mathrm{p}$. 
de l'expression soit au plan du contenu, soit aux deux simultanément, et qui unissent en outre des éléments intra-textuels et des éléments extra-textuels.

Le repérage d'un translème peut résulter d'une lecture tabulaire du texte selon le sens qu'en donne le Groupe Mu dans Rhétorique de la poésie :

Ce type de lecture [...] est le produit de la reconnaissance de plusieurs isotopies et des réévaluations qui autorisent le passage de l'une à l'autre.

Le lecteur de poèmes est donc celui qui peut parcourir le texte selon plusieurs plans de déchiffrement [...] à travers le processus de réactivation du sens?.

Dans cette perspective, contrairement à l'unité conventionnelle de traduction, qui suit la linéarité du texte, le translème est une unité qui peut être transphrastique, voire transtextuelle lorsque le texte s'inscrit dans une interdiscursivité ou une intertextualité. Nous en avons une manifestation dans ce poème qui renferme certains invariants conventionnels du genre de la ballade (tétramètre allitéré, isotopies du voyage, de la mort, personnages à caractère allégorique ou stéréotypés comme le cavalier et le pélerin, etc.) dont l'ensemble constitue un translème intertextuel ${ }^{10}$.

Le découpage du texte en translèmes s'applique particulèrement aux textes qui se présentent comme systèmes modélisants secondaires. Appliqué à l'œuvre littéraire, ce concept de Lotman désigne des textes où le rapport entre les signes de la langue est modifié dans un acte d'énonciation par une série d'opérations qui relèvent principalement de la référentialité, de l'axiologie, de l'esthétique et de l'intertextualité, pour aboutir à la production d'un modèle individué du réel ${ }^{11}$. Etant donné la polyvalence sémantique des constituants d'un texte ainsi défini, puisque ces constituants signifient simultanément mais différemment, et dans le système de la langue, et dans le système du texte, un même élément peut appartenir à plusieurs translèmes. Autrement dit, les translèmes s'interpénètrent ou s'emboîtent les uns dans les autres.

Le repérage d'un translème peut être considéré comme une opération en trois temps, comportant :

1. le repérage des éléments qui composent ce translème ;

2. le repérage des rapports qui existent entre des éléments;

3. le repérage des interférences entre ce translème et les autres.

Pour en donner une illustration, je reprendrai l'exemple des actants. Ils forment à l'intérieur du texte un sous-ensemble sémiotique dont la structuration phonosémantique, binaire et ternaire, permet d'établir un lien logique entre les séquences narratives du poème et, par conséquent, d'en transcender l'hétérogénéité dénotative. L'ensemble des actants constitue donc un translème. La signification de cet ensemble est le produit des rapports phonosémantiques qui unissent ses constituants et que l'on peut figurer à l'aide des deux schémas suivants :

9. Groupe Mu (1977) : Rhétorique de la poésie, Bruxelles, Editions Complexe, p. 163. Ce point de vue du théoricien fait écho à une conception de l'écriture que Michel Butor exprime en ces termes : «... le livre devient un lieu à l'intérieur duquel on se promène. Il y a des livres qui sont si l'on veut, des tunnels ou des rails de chemin de fer, dans lesquels on ne peut faire qu'un seul trajet. Mais j'aime de plus en plus les livres à l'intérieur desquels on peut se promener. Et j'essaie de faire des livres à l'intérieur desquels on peut faire plusieurs trajets différents. Des livres où il y a des cachettes, où l'on va pouvoir trouver des choses peu à peu. " Entrevue avec Michel Butor, le Devoir, 15 août 1981.

10. On trouvera une étude plus approfondie de cet aspect du texte dans mon article $:$ "Intertextualité et traduction. Un poème de W.H. Auden ", dans le Journal canadien de recherche sémiotique / The Canadian Journal of Research in Semiotics, vol. VIII, $n^{\circ} 1$ et 2, automne-hiver 1980-1981, pp. 201-213.

11. Cf. note 2. 


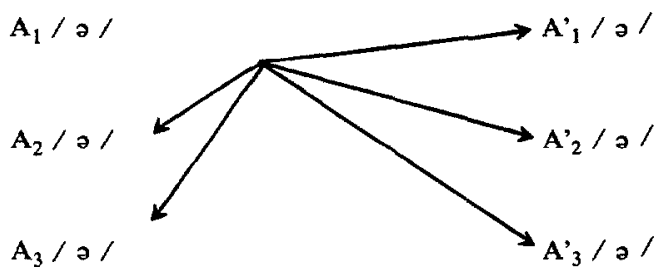

Fig. 1

$$
\downarrow^{P_{s}}
$$

A: actant statique

A': actant dynamique

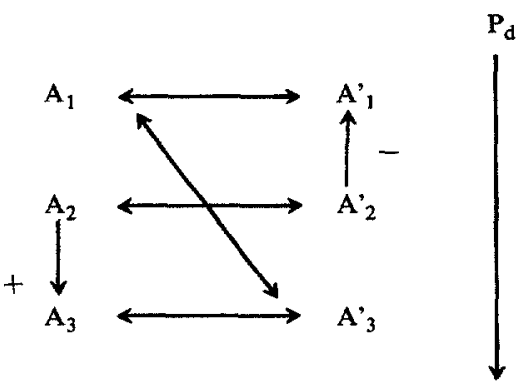

$\mathbf{P}_{\mathbf{s}}$ : paradigme du statisme

$\mathbf{P}_{\mathrm{d}}$ : paradigme du dynamisme

Fig. 2

La densité et la complexité des liaisons phonosémantiques qui existent entre les actants pose au traducteur un problème d'une extrême difficulté. La figure 1 illustre un premier rapport actualisé dans la suffixation agentive et qui relie chaque terme aux cinq autres. Le traducteur doit en outre restituer les rapports illustrés par la figure 2, à savoir :

- la série des trois paronomases, compte tenu du fait que ce dédoublement phonétique réalise une liaison sémantique paradoxale qui renvoie à la dialectique du même et du contraire ou du complémentaire ;

- l'opposition biparadigmatique du statisme $\left(P_{s}\right)$ et du dynamisme $\left(P_{d}\right)$; digme.

- le rapport d'intensité, croissante ou décroissante, à l'intérieur de chaque para-

Il doit aussi maintenir explicitement le terme 'lecteur' au début de la série de même que son interchangeabilité avec le dernier terme de cette série car, outre sa fonction herméneutique, cette liaison installe les trois premières strophes dans une circularité et une répétitivité qui iconisent le discours des actants statiques où s'exprime la pulsion de mort.

À ces contraintes s'ajoutent celles qu'impose le système du texte, et c'est là que jouent les interférences entre les translèmes. Par exemple, chacun des actants est simultanément indexé sur une ou plusieurs isotopies, notamment celle du voyage et celle de la mort (auxquelles se rattachent explicitement 'where', 'going', 'valley', 'yonder', ' return', etc., et 'fatal', 'grave', etc.), entrant ainsi dans la composition d'autres translèmes. De plus, chacun des actants a une importance dans le système axiologique du poème. Ainsi, rendre la paronomase 'reader" / 'rider' par 'lecteur' / 'licteur' entraîne la destruction du système de valeurs du texte et, par conséquent, du code qui permet de déchiffrer le message. 
Il en résulte que le texte poétique, en tant que système modélisant secondaire, est un texte surcodé. Ce surcodage, qui est à la source de la polyvalence sémiotique de ses éléments, entraîne une surtraduisibilité de ce texte. Autrement dit, le texte poétique ne peut être idéalement restitué comme totalité signifiante que par la "somme de ses traductions significativement différentes » (la formule est de Léon Robel) ${ }^{12}$, mais il est difficile, voire impossible, d'en rendre la simultanéité dans la langue cible. La traduction du texte poétique procède donc nécessairement d'une hiérarchisation des translèmes à l'intérieur d'un programme translatif.

Encore faut-il bien voir que dans cette hiérachisation, tout comme dans l'établissement du programme translatif qui la précède, intervient un sujet à la fois individuel et social. Celui-ci est défini par un ensemble de coordonnées historiques qui, sur le plan axiologique, idéologique et esthétique, limitent ou bloquent par avance l'ouverture du texte tout autant que celle de sa traduction.

12. Léon Robel (1973) : "Translatives ", Changes 14, Paris Seuil, p. 8. 